\title{
La valeur et le prix
}

\section{Samia Hurst}

Prof. Dr méd., Institut Ethique Histoire Humanités (iEH2), Faculté de médecine, Genève, membre de la rédaction

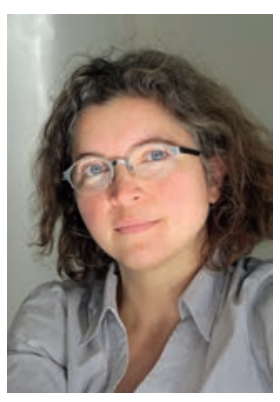

Pour ne pas payer plus cher, que sommes-nous prêts à sacrifier? Cette question a malheureusement pris une actualité effrayante avec l'incendie de la tour Grenfell à Londres le 14 juin dernier. L'incendie, qui a fait près de 80 morts, aurait été accéléré en partie par un revêtement connu pour être inflammable. Il était légèrement moins cher que le revêtement concurrent. Très légèrement: l'économie totale, pour le bâtiment entier, aurait été d'environ 5000 £. On comprend la rage des survivants.

Economiser de l'argent, une bonne chose à la base pourtant, cela devient problématique lorsque l'on sacrifie des choses qui auraient largement valu la dépense supplémentaire. Cette petite observation - banale au fond - aurait des implications profondes si nous la prenions véritablement au sérieux. Car ce que nous sacrifions pour économiser de l'argent, il arrive que ce soit invisible. Et si les habitants de la Grenfell Tower auraient dû être visibles pour les propriétaires, il arrive aussi que cette invisibilité soit plus compréhensible. C'est à ce propos que ma collègue Annette Rid a récemment signé un article très intéressant sur les analyses d'impact budgétaire [1]. Vous vous rappelez la décision sur le remboursement du Sofosbuvir contre l'hépa-

\section{Economiser de l'argent, une bonne chose}

à la base pourtant, cela devient problématique lorsque l'on sacrifie des choses qui auraient largement valu la dépense supplémentaire.

tite C? C'est un médicament clairement efficace, et dont l'analyse coût-efficacité montre que le rapport qualité-prix est dans les marges généralement acceptées en Suisse [2]. Seulement voilà: le nombre de patients concernés rendrait la facture totale insoutenable. Il n'est donc remboursé que pour les patients les plus atteints. C'est cela, l'analyse d'impact budgétaire.
Contrairement à l'histoire de l'incendie londonien, cette décision-ci commence par paraître très raisonnable. Si nous n'avons pas les moyens, c'est ainsi. Le critère de priorité aux plus atteints est même plutôt juste: ce sont eux qui verront la plus grande différence, eux également qui ne peuvent pas attendre. Et pourtant, ici aussi on sacrifie des choses inaperçues. Refuser un traitement en raison du nombre de patients atteints introduit une inégalité de traitement flagrante entre patients: certains seront moins bien soignés du simple fait que leur groupe est plus nombreux. On sacrifie une part d'équité. Refuser la prise en charge transforme ces patients en otages dans la négociation qui opposent les vendeurs et les états: un problème moral où les uns et les autres ont leur part, certes, mais un problème tout de même. On sacrifie une part de leurs droits. Finalement, limiter l'accès à un traitement dont l'impact budgétaire est élevé suppose qu'il serait impossible de mobiliser cet argent en choisissant de limiter d'autres prestations moins efficaces ailleurs. On sacrifie ici de l'efficience, une part du bien que l'on pourrait faire avec les moyens disponibles. Que faire alors? Pas de recette miracle à ce stade. Pourrions-nous nous doter d'un mécanisme plus systématique pour décider ce que nous voulons retirer du budget, lorsque survient une situation de ce type? A y bien regarder, peut-être même que nous déciderions alors de ne pas faire ces sacrifices? Ce ne serait certainement pas simple, mais au moins nous aurions alors les valeurs sous les yeux et pas seulement le prix.

\section{Références}

1 Charlton V, Littlejohns P, Kieslich K, Mitchell P, Rumbold B, Weale A, et al. Cost effective but unaffordable: an emerging challenge for health systems. Bmj. 2017 Mar 22;356:j1402. PubMed PMID: 28330879.

2 Pfeil AM, Reich O, Guerra IM, Cure S, Negro F, Mullhaupt B, et al. Cost-effectiveness analysis of sofosbuvir compared to current standard treatment in Swiss patients with chronic hepatitis C. PloS one. 2015;10(5):e0126984. PubMed PMID: 25974722. Pubmed Central PMCID: 4431849. 